\title{
Retraction Note to: Relationships between genetic polymorphisms in inflammation-related factor gene and the pathogenesis of nasopharyngeal cancer
}

\author{
Yan-Li Qu ${ }^{1,2}$ • Hong Yu ${ }^{2}$ - Yan-Zhi Chen ${ }^{1} \cdot$ Yu-Xia Zhao ${ }^{1}$ - Guang-Jun Chen ${ }^{3}$. \\ $\mathrm{Lu} \mathrm{Bai}^{4} \cdot$ Dan Liu ${ }^{1} \cdot$ Hong-Xin Su${ }^{1} \cdot$ He-Tong Wang ${ }^{1}$
}

Published online: 17 August 2015

(C) International Society of Oncology and BioMarkers (ISOBM) 2015

Retraction to: Tumor Biol. (2014) 35:9411-9418

DOI 10.1007/s13277-014-2123-6

The Publisher and Editor retract this article in accordance with the recommendations of the Committee on Publication Ethics (COPE). After a thorough investigation we have strong reason to believe that the peer review process was compromised.

The online version of the original article can be found at http://dx.doi.org/ 10.1007/s13277-014-2123-6.

Yu-Xia Zhao

cmu4h_zyx@yeah.net

1 Department of Radiation Oncology, Fourth Affiliated Hospital of China Medical University, Chongshan Earth Street Number 4, Huanggu District, Shenyang 110032, China

2 Department of Radiation Oncology, Liaoning Cancer Hospital and Institute, Shenyang, Liaoning, China

3 Clinic of Border Control Bureau of Liaoning Province, Shenyang, Liaoning, China

4 Department of Radiation Oncology, First Affiliated Hospital of China Medical University, Shenyang, China 